\title{
Detection of Cotton Maturity and Skin Defects based on Photoelectric Detection Technology
}

\author{
Hui Zhang ${ }^{1, \text { a }}$ \\ ${ }^{1}$ Changchun University of Science and Technology, Changchun, Jilin, 130022 \\ a email
}

Keywords: Cotton Maturity, Skin Defects, Photoelectric Detection

\begin{abstract}
Cotton fiber maturity and fineness parameters are an important index affecting the quality of textile processing. The indirect measurement method can not accurately evaluate the quality and quality of cotton fiber.In this paper, the Fraunhofer diffraction optical method and linear array CCD are used to detect cotton fiber diffraction $\left(23{ }^{\circ} \mathrm{C}\right)$ and constant humidity $(45 \%)$, and the cotton grades with different maturity grades were obtained in the laboratory temperature $\left(23{ }^{\circ} \mathrm{C}\right)$ and constant humidity (45\%), and the cotton fiber was measured by wavelet analysis. The results show that the two methods have a good correlation, the correlation coefficient is 0.956 , the mean square error is 0.332.Therefore, based on the photoelectric detection technology method can realize the rapid detection of cotton fiber maturity in the laboratory, For the actual production of cotton maturity directly, fast photoelectric detection provides a theory.
\end{abstract}

\section{Introduction}

Cotton fiber maturity and fineness parameters are an important indicator of the quality of textile processing, but in the cotton circulation, production and processing of this parameter is difficult to ensure the accuracy of the test, the current maturity of cotton commonly used detection methods are Two methods: direct measurement method and indirect measurement method, among them indirect measurement methods are micronaire, dyeing and near infrared measurement, direct measurement methods are high accuracy microscope and high accuracy camera, etc. Cotton maturity and the direct distribution of cotton fibers is the direct relationship between the diameter distribution of cotton fibers (the size of the cross-section of cotton fibers, the number of crimps, etc.) The direct detection method is the basis of other detection methods, usually in the laboratory through the high power microscope, Sectional shape, according to the cotton fiber maturity and cotton fiber cross-sectional shape relationship, accurate assessment of the maturity of cotton samples.

Lint defect is the fiber with impurities and hinder the textile fiber two categories. According to GB1103-2007 "cotton fine cotton" standard requirements, defects include: seeds, infertility seeds, cable silk, soft seed epidermis, stiff film, with fiber seed and cotton knot 7 kinds. As the defects in the textile process is difficult to discharge, residual impurities in the yarn wrapped in the yarn or attached to the surface of the yarn, the consequences are caused by the deterioration of the yarn, yarn twist, impurities increased, yarn wool feathers increased. With a large amount of fiber with a large number of yarns caused by the surface of the embryo cloth showed a lot of neps, impurities, dyeing after the fabric will appear infertility color, wool, feel stiff and rough, thus affecting the development of cotton textile industry, to the national economy has brought serious losses. Therefore, the rapid detection of lint defect content is essential for determining the quality of raw cotton. At present, most enterprises in the country generally use visual inspection of defects. Visual test accuracy is often affected by human factors, but also for small defects, the detection results of false detection rate and missed rate is relatively high, and time-consuming. With the development of computer technology and photoelectric detection technology, machine vision technology has been widely used in product defects and defect detection. Application of visible light machine vision is infrared band spectral image and tomography and other detection technology, the use of digital image processing and stoichiometric analysis method to identify the classification of raw cotton impurities. In the image processing technology, it is very important to divide the lint defect image 
accurately. Li Meiling et al. Proposed a method to combine the maximum interclass variance method and linear regression to realize the recognition of knot. Li Guohui et al. Proposed a kind of cotton white heterosexuality based on irregular imaging machine vision system Image segmentation method for fiber detection.

\section{Measurement System}

Cotton Inspection Microscope is a new instrument produced by the Australian Cotton Inspection Microscope, which can measure the fineness and maturity of cotton fiber directly. It is a direct, basic and precise method and fast ( $<30 \mathrm{~s} /$ times, excluding short fibers Sample preparation). This means that the measurement results obtained by the instrument are accurate and that the speed of the instrument means that the accuracy of the measured value reaches the level acceptable to the market. Figure 2 shows the picture of the instrument and the field of view. The cotton microscope uses a conventional birefringence method to measure the fiber maturity and the length of the fiber mass of known mass, thereby measuring the fineness or line density of the fiber. The technical breakthrough of the cotton inspection microscope includes placing the short fiber sample in a measuring tank filled with water so that the staple fibers are randomly dispersed in the measurement; the coupled polarized light is transmitted through the water system to obtain a very clear fiber image; the improved illumination And the optical system enables the captured fibers to be clearly shown, and the application of special image processing techniques and data analysis algorithms can accurately introduce the maturity and fineness of the sample. Cotton inspection microscopes can complete 20000 fiber segments within $30 \mathrm{~s}$, and this technology is well suited for commercialization. The reference values of these cotton were measured using a recognized direct method, that is, cross section sections of cotton fibers, to observe the cross-sectional geometry, cell wall thickness (maturity). The initial laboratory test performance of the cotton microscope was as follows: the maturation rate and fineness of the mixed cotton fiber samples ( 5 replicates) were 0 . $2 \%$ and 1 . The correlation coefficient (R2) between the maturation rate and the linear density measured by a cotton microscope was different from that of the Texas Tech University, but covered its maximum and minimum ranges, with a maturity ratio exceeding $85 \%$, line density of more than 91\%. The measured values between the instruments were well reproducible, with reference to cotton from the Texas Technical University as a sample, and the correlation coefficient between the three different cotton microscopy microscopes was over $99 \%$.

The experimental device consists of four parts: the sample test bed, the light source, the image acquisition unit and the data processing unit. In order to avoid stray light interference, improve the detection accuracy, sample test bench, light source and image acquisition part installed in the darkroom. The light source is composed of two $9 \times 11$ white light emitting diode (LED) arrays, oblique $45^{\circ}$ on the sample to be tested, an area of $8 \mathrm{~cm} \times 25 \mathrm{~cm}, 20 \mathrm{~cm}$ from the test sample height. In the experiment, the lint samples with uniform defects and different positions were prepared by hand, and the samples were sagged with smooth and smooth glass. The samples were $18 \mathrm{~cm} \times 20$ $\mathrm{cm} \times 2 \mathrm{~cm}$ in length and width. Resolution of 640 pixel $\times 480$ pixel digital camera fixed above the test platform to collect the sample color samples, $25 \mathrm{~cm}$ away from the sample to be measured, the maximum size of cotton samples collected $16 \mathrm{~cm} \times 18 \mathrm{~cm}$. The image is transferred to the terminal data processing unit via USB data cable. The processor operating system is Windows 2007, and the image processing is analyzed by Matlab7.0. He is a color image of the lint samples containing various defects collected by the experimental device. Due to the non-uniformity of the complementary metal-oxide semiconductor (CMOS) sensitivity during the photoelectric conversion process, the quantization noise of the digital process and the channel error of the transmission process the image has a certain degree of noise interference. Noise deteriorates the image quality and brings difficulties to the later analysis. Therefore, it is necessary to preprocess the image with gray scale correction and noise filtering. Study flow chart of raw cotton defect detection algorithm.

The traditional integrated edge detection operator is based on the average of the three-channel edge strength, and the edge strength of each defect of cotton is strengthened at the same time, which is not conducive to the identification of cotton defects. Zhang Xin, etc. Using the square root of the 
square sum of the three-channel edge strength as the integrated edge strength, this method has better effect on cotton heterosexual fiber detection, but the detection effect of defect is poor. The results of manual detection and system detection were compared, and the defects were measured as broken seeds, with fiber seed and frosted cotton. From the experimental results, it can be seen that the correct rate of raw cotton defect is more than $85 \%$, the detection time is less than $3 \mathrm{~s}$, the detection time is related to the content of raw cotton defect, and the stability of the detection system is good, which can meet the requirement of real-time detection.

\section{Cotton Maturity Measurement System}

The light source is a helium-neon laser 1 (Thorlabs HRP008) capable of emitting a polarized laser beam with a power of $0.8 \mathrm{~mW}$ and a wavelength of $632.8 \mathrm{~nm}$. The diaphragm 2 can remove the stray light around the laser spot so that the beam brightness on the cotton fiber is even more uniform, the polarizer 3 can adjust the size of the incident laser light and remove the stray light interference. The single cotton fiber 4 is fixed on the one-dimensional precision optical frame (Newport420) and the focal lens of the focal length of $100 \mathrm{~mm}$ by the cotton fiber clip 5 (ThorlabsLB1676) The diffractive signal is converged on the linear array detector 8 (Mightex TCN-1304-U), and the light-blocking sheet 7 in front of the detector avoids the interference of the zero-order diffraction pattern to the diffraction signal.

In the experiment, the cotton fiber was slightly straightened and the precision optical frame was rotated. Each time the 1-inch linear array CCD was used to detect the diffraction pattern of the corresponding position of the cotton fiber. The diffraction signal was transferred to the microcomputer through USB and subjected to wavelet analysis denoising. The diameter of cotton fiber was calculated according to the relationship between oblique lines and filament diameter. According to the multiple measuring points, the fiber diameter distribution of single cotton fiber was obtained, and the results were basically consistent with the microscope (Olympus BX41).

In the experiment, the cotton samples of eight different varieties were measured at constant temperature $\left(23{ }^{\circ} \mathrm{C}\right)$ and constant humidity (45\%), and the cotton samples were tested by high capacity tester (HVI). The photoelectric detection devices and the correlation coefficient between the two methods was 0.956 and the mean square error was 0.332 . The results showed that the photoelectric detection device was basically consistent with the microscopic measurement of cotton fiber diameter distribution, and the photoelectric detection device was measured. The results are slightly larger than the microscopic measurement results, because the results based on Fraunhofer diffraction photodetection are too large, so the calculation formula should be modified at the later stage.Figure 3 The two kinds of measurement methods are used to measure the diameter of the same root fiber The error of the two measurement methods is mainly due to the fact that the position of the microscopic measurement sample does not exactly correspond to the position of the sample measured by the photoelectric detection device.

The maturity and fineness parameters of cotton fiber are an important index which affects the quality of textile processing. However, it is difficult to ensure the accuracy of this parameter in the circulation, production and processing of cotton. The results are often very different. The results show that the two methods have good correlation coefficient, the correlation coefficient is 0.956 and the mean square error is 0.332 . Therefore, the photoelectric detection technology can accurately measure the diameter distribution of cotton fiber in the laboratory, which provides a basis for the direct and rapid detection of cotton maturity, and has important theoretical significance.

\section{Conclusion}

A fast detection method based on improved adaptive iterative threshold method for lint defect was proposed for seedling, banding and stiffening of lint defect. Based on the photoelectric detection technique, the edge detection of the multi-defect image is carried out by using the improved morphological edge detection operator. The defect image with obvious characteristic is obtained, and the threshold is used to calculate the threshold image. The detection rate of raw cotton defect is 
more than $85 \%$, and the detection time is less than $3 \mathrm{~s}$, which can meet the requirement of rapid detection.

\section{References}

[1] Su Junhong, Liu Shengli. Surface Defect Detection and Morphology Analysis of Cylindrical High Precision Parts[J]. Advances in Laser and Optoelectronics. 2014 (04)

[2] Wang Yanfei, Wang Cunyue, Xu Wenji. Study on laser cutting quality of aluminum alloy sheet based on image processing [J]. China Laser. 2014 (01)

[3] Yao Hongbing Study on Recognition of Lens Flaw Type Based on Image Processing [J]. Progress in Laser and Optoelectronics 2013 (11)

[4] Gao Yan, Shao Shuangyun, Feng Qibo. A method for automatic measurement of geometric parameters of wheel pairs by laser scanning [J]. Chinese Journal of Laser Science. 2013 (07)

[5] Zhang Zhifeng.Study on rapid assessment method of cotton maturity based on photoelectric detection technology[J]. Acta Photonica Sinica 2011 (03) 